associated with 21 genes including plasma cell (CD38), T cell (CD4), co-stimulation (CD80/86/CTLA4), IL-7 as lymphoid tissue inducer, TNF $\alpha$ and STAT-1/STAT-5 signalling, CCL21 and IgG/IgM heavychains. No difference was observed for the expression of CD68 (macrophages) between diseases, levels of inflammation or TA. CD68 expression was related to TNF $\alpha$, TGF $\beta$ and CD86. CD4 (T cells) was related to IL-6 and STAT5 (rho=0.768, p<0.001). CD20 (B cells) was hardly detected but CD38 (plasma cells) were related to the expression of BLIMP1 (rho=0.900, p<0.001), IKZF-3, CD79, IgM/IgG heavychains, Ig constant-chain and STAT-1.

Conclusion Altogether, these data suggest that analysis of gene expression using qPCR in synovial biopsy is technically feasible with limited data loss. The analysis of gene expression levels however needs to be closely examined in relation to the expression of cell lineage markers as it is clear that the cellular composition of the biopsy will impact on analysis.

\title{
14 PILOT GENE EXPRESSION ANALYSIS OF INFLAMED SYNOVIUM
}

F Ponchel, S M Churchman, V Goeb, E A Horner, K Henshaw, P Emery Division of Musculoskeletal Disease, Leeds Institute of Molecular Medicine, University of Leeds, and the NIHR Leeds Musculoskeletal Biomedical Research Unit, Leeds Teaching Hospital NHS Trust, Leeds, UK

\subsection{6/annrheumdis-2011-201239.14}

Background and objectives Within the synovial membrane of an rheumatoid arthritis (RA) joint, tissue architecture varies depending on organisation of the infiltrating inflammatory cells (diffuse, aggregates and germinal centres). It has been hypothesised that more organised cell network could represent a true autoimmune phenotype due to better presentation of auto-antigens and higher expression of various cytokines and chemokines, leading to the hypothesis that these differing tissue architectures (TA) could predict response to biologics. The authors designed a customised gene array to pilot the feasibility of gene expression signatures in synovial biopsy.

Methods Twenty biopsies from 12 RA and 8 OA patients were collected by arthroscopy with scored joint inflammation (VAS). Tissue architecture was classified in three categories; diffuse infiltration, aggregates and germinal centre-like structures. A custom 96 gene Taqman array was designed (including three reference genes) and expression was measured using quantitative real-time (q)PCR.

Results Of the 93 genes tested, only 1 was below detection and 3 undetected in over $75 \%$ of samples. Pilot analysis was performed as the number of samples was not allowing highly significant difference to be found. Analysing samples based on diagnostic criteria showed differential expression of 25 genes between RA and OA including TNF $\alpha$ but not IL-1/6/10/17/18, CD4 ( $T$ cell) $(p=0.064)$, but not B/plasma cell lineage markers (CD20/38/138), increased co-stimulation (CTLA4/CD86), chemokines (CXCL13/CCL12), PADI4 for citrullination and HDAC7 for epigenetic modification. Expression of 21 genes was related to local levels of inflammation: CCL21, Ig constant chain and IgG/IgM heavy chains, chemokine CXCL13 and BLIMP1 (rho $=0.753, \mathrm{p}<0.001$ ) a transcription factor involved in $B$ cell differentiation. Increasing TA complexity was 